\title{
EXISTENCE OF WEAK SOLUTIONS FOR ABSTRACT HYPERBOLIC-PARABOLIC EQUATIONS
}

\author{
MARCONDES RODRIGUES CLARK \\ Universidade Federal da Paraíba - Campus II - DME - CCT \\ 58.109-970 - Campina Grande - Paraíba - Brasil \\ e-mail:mclark at brufpb2.bitnet \\ (Received July 30, 1992 and in revised form April 19, 1993)
}

\section{ABSTRACT}

In this paper we study the Existence and Uniqueness of solutions for the following Cauchy problem:

$$
\begin{aligned}
& A_{2} u^{\prime \prime}(t)+A_{1} u^{\prime}(t)+A(t) u(t)+M(u(t))=f(t), \quad t \in(0, T) \\
& u(0)=u_{0} ; A_{2} u^{\prime}(0)=A_{2}^{\frac{1}{2}} u_{1} ;
\end{aligned}
$$

where $A_{1}$ and $A_{2}$ are bounded linear operators in a Hilbert space $H,\{A(t)\}_{0 \leq t \leq T}$ is a family of self-adjoint operators, $M$ is a non-linear map on $H$ and $f$ is a function from $(0, T)$ with values in $H$.

As an application of problem (1) we consider the following Cauchy problem:

$$
\begin{aligned}
& k_{2}(x) u^{\prime \prime}+k_{1}(x) u^{\prime}+A(t) u+u^{3}=f(t) \text { in } Q, \\
& u(0)=u_{0} ; k_{2}(x) u^{\prime}(0)=k_{2}(x)^{\frac{1}{2}} u_{1}
\end{aligned}
$$

where $Q$ is a cylindrical domain in $\mathbb{R}^{4} ; k_{1}$ and $k_{2}$ are bounded functions defined in an open bounded set $\Omega \subset \mathbb{R}^{3}$,

$$
A(t)=-\sum_{i, j=1}^{n} \frac{\partial}{\partial x_{j}}\left(a_{i j}(x, t) \frac{\partial}{\partial x_{i}}\right) ;
$$

where $a_{i j}$ and $a_{i j}^{\prime}=\frac{\partial}{\partial t} u_{i j}$ are bounded functions on $\Omega$ and $f$ is a function from $(0, T)$ with values in $L^{2}(\Omega)$.

KEY WORDS AND PHRASES: Existence of weak solutions, Nonlinear equation, Cauchy problem, Existence and Uniqueness.

\section{AMS Subject Classifications:35L15}

\section{INTRODUCTION}

Let $T>0$ be a positive real number and $\Omega$ be a bounded open set of $\mathbb{R}^{n}$, with smooth boundary $\Gamma$. In the cylinder $Q=\Omega \times(0, T)$, Bensoussan et al. [01], studied the homogeneization for the following Cauchy problem:

$$
\begin{aligned}
& k_{2}(x) u^{\prime \prime}+k_{1}(x) u^{\prime}-\Delta u=f \text { in } Q . \\
& u(x, 0)=u_{0}(x) \text { e } k_{2}(x) u^{\prime}(x, 0)=k^{\frac{1}{2}} 2(x) u_{1}(x), \quad x \in \Omega
\end{aligned}
$$

Many authors have been investigating the existence of solution for non-linear equations associated with problem (3),

see: Larkin [04], Lima [05], Medeiros [07-09], Melo [10], Maciel [11], Neves [12] and Vagrov [15].

Other interesting results relative to existence of a solution for a non-linear equation associated with the equation of the problem (3) can be found in the work of J'orgens [03] 
In this is work he proved the existence of classical solution by iterative methods for the mixed problem associated to the equation

$$
u_{t t}-\Delta u+F^{\prime}\left(|u|^{2}\right) u=0
$$

in open domain of $\mathbb{R}^{3}$, with the hypotesis $F(0)=0$ and $\left|F^{\prime}(s)\right| \leq a[b+F(s)]^{\alpha}$ where $a, b$ and $\alpha$ are positive constants with $\alpha<\frac{2}{3}$.

In Section 1, we establish some notation for the function spaces and conditions for $A_{1}, A_{2},\{A(t)\}_{0 \leq t \leq T}, M$ and $f$ in equation (1). In Section 2, we state our main results and we prove the assertions made. In the final Section we make an application of problem (1).

\section{PRELIMINARIES}

We will assume that standard function spaces are known: $C^{k}(\Omega), L^{p}(Q) ; H^{k}(\Omega)$, $H_{0}^{k}(\Omega), C^{k}(0, T ; X), L^{p}(0, T ; X)$ where $X$ is a Banach space.

Let $H$ be a real Hilbert space, with inner product and the norm denoted by $(\cdot, \cdot)$ and $|\cdot|$, respectively.

We consider here the following assumptions:

i) $A_{2}: H \rightarrow H$, a positive symmetric operator

ii) $A_{1}: H \rightarrow H$, a symmetric operator such that:

$$
\left(A_{1} u, u\right) \geq \beta|u|^{2}, \quad 0<\beta \in \mathbb{R}, \text { for all } u \in H .
$$

iii) Let $\{A(t) ; t \geq 0\}$ be a family of self-adjoint linear operators of $H$, such that there exists a constant $\alpha>0$, satisfying $(A(t) u, u) \geq \alpha|u|^{2}$ for all $u \in D(A(t))$, where we assume that the domain $D(A(t))$ of $A(t)$ is constant, i.e, $D(A(t))=$ $D(A(s)) \forall t, s \geq 0$. It is known from the spectral theory for self-adjoint opeators that there exists only one positive self-adjoint operator $A^{\frac{1}{2}}(t)$ such that:

$$
D(A(t)) \subseteq D\left(A^{\frac{1}{2}}(t)\right)
$$

From assumption iii) we have, see Medeiros [09], that $D\left(A^{\frac{1}{2}}(t)\right)$ is constant.

Let $V_{t}=D\left(A^{\frac{1}{2}}(t)\right)$ with inner product $((\cdot))$ and associated norm $\|\cdot\|_{t}$. Therefore $\|u\|_{t}^{2}=\left|A^{\frac{1}{2}}(t) u\right|^{2} \geq \alpha|u|^{2}$.

So that, $V_{t}$ is a Hilbert space, dense and embedded in $H\left(V_{t} \hookrightarrow H\right)$, and $V_{t}$ is isomorphic with $V_{0}, \forall t$.

iv) $A(t)$ is continuously strongly differentiable.

v) For $u \in D(A(0))$, we assume that there exists a real $\gamma>0$, independent from $t$, such that:

$$
\left(A^{\prime}(t) u, u\right) \leq \gamma\|u\|_{0}^{2}, \quad \forall t \in[0, T]
$$

vi) We assume that the embedding $V_{0} \hookrightarrow H$ is compact. Therefore, the spectrum of the operator $A(t)$ is discret. 
Identifying $H$ with his dual $H^{\prime}$, we have the immersions:

$V_{0} \hookrightarrow H \hookrightarrow V_{0}^{\prime}$; where each space is dense on the following one.

In this work, we use the symbol $\langle\cdot, \cdot\rangle$, to denote the duality between $V_{0}^{\prime}$ and $V_{0}$. Sometimes it means an application of a Vector distribution to a real test function.

vii) Let $M$ be an operator of $V_{0}$ in $H$ satisfying the following conditions:

a) $M$ is monotone, hemi-continuous and bounded (in the sense of taking bounded sets of $V_{0}$ into bounded sets of $H$ ).

b) There exists a constant $\sigma>0$ so that

$$
\int_{0}^{T}\left(M(u(s)), u^{\prime}(s)\right) d s \geq-\sigma \forall t \in\{0, T] \text { and } \forall u \in E_{C}
$$

where $E_{\bar{C}}$ denotes the set $\left\{u \in L^{\infty}\left(0, T ; V_{0}\right) ; u^{\prime} \in L^{2}(0, T ; H)\right.$ and $\left.\|u(0)\|_{0} \leq \bar{C}\right\}$

\subsection{The Main Results}

Theorem - 1: (Existence) Under the above assumptions (i-vii) and considering

$$
\begin{gathered}
f \in L^{2}(0, T ; H) \\
u_{0} \in V_{0} \\
u_{1} \in H,
\end{gathered}
$$

then there exists a function $u$ defined in $(0, T)$ with values in $V_{0}$ such that:

$$
\begin{aligned}
& u \in L^{\infty}\left(0, T ; V_{0}\right) \\
& u^{\prime} \in L^{2}(0, T ; H),
\end{aligned}
$$

besides this, $u$ is a solution of problem (1) in the following way:

$$
\begin{aligned}
& -\int_{0}^{T}\left(A_{2} u^{\prime}(t), \Phi^{\prime}(t) v\right) d t+\int_{0}^{T}\left(A_{1} u^{\prime}(t), \Phi(t) v\right) d t+ \\
& +\int_{0}^{T}\left(A^{\frac{1}{2}}(t) u(t), A^{\frac{1}{2}}(t) \Phi(t) v\right) d t+ \\
& +\int_{0}^{T}(M(u(t)), \Phi(t) v) d t=\int_{0}^{T}(f(t), \Phi(t) v) d t, \quad \forall v \in V_{0}
\end{aligned}
$$

and $\forall \Phi \in C_{0}^{1}(0, T)$.

$$
\begin{gathered}
u(0)=u_{0} \\
A_{2} u^{\prime}(0)=A_{2}^{\frac{1}{2}} u_{1} .
\end{gathered}
$$

For the uniqueness we need the following condition on $M$ :

viii) Given $C>0$, there exists $K>0$, which depends on $C$, such that:

$$
|M(u)-M(v)| \leq K|u-v|
$$

for all $u, v \in V$ whenever $\|u\|_{0} \leq C$ and $\|v\|_{0} \leq C$. 
Theorem - 2. (Uniqueness) Suppose that the operators $A_{1}, A_{2}, A(t)$ satisfy the conditions of Theorem-1 and (viii), respectively, and $M$ maps functions of $L^{\infty}\left(0, T ; V_{0}\right)$ into functions of $L^{2}(0, T ; H)$. Then, there exists at most one function $u$ in the class

$$
u \in L^{\infty}\left(0, T ; V_{0}\right), u^{\prime} \in L^{2}(0, T ; H),
$$

and $u$ is a solution of problem (1) in the sense (2.6) - (2.8) of Theorem-1.

\section{Remark 2.1}

From (2.4), (2.5) and (2.6) we obtain that $A_{2} u^{\prime \prime} \in L^{2}\left(0, T ; V_{0}^{\prime}\right)$ and this together with (2.4) (2.5) imply that the initial conditions (2.7) (2.8) make sense.

\subsection{Proof of the Theorems}

In this part we use the followin result:

Lema 1. Let $u \in L^{2}(0, T ; H), u^{\prime} \in L^{2}\left(0, T ; V_{0}^{\prime}\right)$ with $v$, and $v^{\prime} \in L^{2}\left(0, T ; V_{0}\right)$. Then

$$
\frac{d}{d t}<u, v>=<u^{\prime}, v>+\left(u, v^{\prime}\right)
$$

For the proof of this lemma see Tanabe, [13].

We apply the standard Galerking approximate procedure. Let $\left(w_{\nu}\right)$ be a base of $D(A(0))$ that it is a base of $H$, by density. From the assumption (i), we have $\left(\left(A_{2}+\right.\right.$ $\left.\lambda I)^{\frac{1}{2}} w_{\nu}\right)$ is also a base of $H$; where $\lambda>0$ is a constant. Let $V_{m}(0)$ be a subspace of $D(A(0))$ generated by the first- $m$ vectors $w_{1}, \ldots, w_{m}$, and $V_{m}^{\lambda}(0)$ the subspace generated by first- $m$ vectors $\left(A_{2}+\lambda I\right)^{\frac{1}{2}} w_{1}, \ldots,\left(A_{2}+\lambda I\right)^{\frac{1}{2}} w_{m}$.

We put $u_{\lambda m}(t)=\sum_{i=1}^{m} g_{\imath \lambda} m(t) w_{\imath}$ as a solution of the approximate perturbed problem:

$$
\begin{aligned}
& \left(\left(A_{2}+\lambda I\right) u_{\lambda m}^{\prime \prime}(t)+A_{1} u_{\lambda m}^{\prime}(t)+A(t) u_{\lambda m}(t)+\left(M\left(u_{\lambda m}(t)\right), v\right)=\right. \\
& =(f(t), v), \quad \forall v \in V_{m}(0) . \\
& \quad u_{\lambda m}(0)=u_{0 m} ; \text { where } u_{0 m}=\sum_{i=1}^{m} \alpha_{i m} w_{i} \rightarrow u_{0}
\end{aligned}
$$

strongly in $V_{0}$

$$
u_{\lambda m}^{\prime}(0)=u_{1 \lambda m} ; \quad \text { where } u_{1 \lambda m}=\sum_{i=1}^{m} \beta_{i \lambda m} w_{i}
$$

where the coefficient $\beta_{\imath \lambda m}$ denotes the coordinates of the vector $P_{\lambda m} u_{1}$, the orthogonal projection of the vector $u_{1}$ upon the subspace $V_{m}^{\lambda}(0)$ in relation to the base $\left(\left(A_{2}+\lambda I\right)^{\frac{1}{2}} w_{\nu}\right)$, such that:

$$
P_{\lambda m} u_{1}=\sum_{i=1}^{m} \beta_{\imath \lambda m}\left(A_{2}+\lambda I\right)^{\frac{1}{2}} w_{i} .
$$

We have that $P_{\lambda m} u_{1} \rightarrow u_{1}$ strongly in $H$ and satisfies

$$
\left|P_{\lambda m} u_{1}\right| \leq\left|u_{1}\right| \forall m \text { e } \forall \lambda>0 .
$$

System (2.9) - (2.11) is equivalent to a system of non-linear ordinary differential equations, which has a solution $u_{\lambda m}(t)$ by using Caratheodory's theorem ,see Coddington - Levinson [02]; defined in an interval $\left[0, t_{m}\right)$, with $t_{m}<T$, for each $m \in \mathbb{N}$. 


\section{3 - "A priori" Estimates}

In (2.9) taking $v=2 u_{\lambda m}^{\prime}(t)$ we have:

$$
\begin{aligned}
& \frac{d}{d t}\left|\left(A_{2}+\lambda I\right)^{\frac{1}{2}} u_{\lambda m}^{\prime}(t)\right|^{2}+2\left(A_{1} u_{\lambda m}^{\prime}(t), u_{\lambda m}^{\prime}(t)\right)+ \\
+ & 2\left(A^{\frac{1}{2}}(t) u_{\lambda m}(t), A^{\frac{1}{2}}(t) u_{\lambda m}^{\prime}(t)\right)+2\left(M\left(u_{\lambda m}(t)\right), u_{\lambda m}^{\prime}(t)\right)= \\
= & 2\left(f(t), u_{\lambda m}^{\prime}(t)\right) .
\end{aligned}
$$

Using the above assumptions, we have,

$$
\begin{aligned}
& \left|\left(A_{2}+\lambda I\right)^{\frac{1}{2}} u_{\lambda m}^{\prime}(t)\right|^{2}+\beta \int_{0}^{t}\left|u_{\lambda m}^{\prime}(s)\right|^{2} d s+\left\|u_{\lambda m}(t)\right\|_{t}^{2} \leq \\
\leq & 2 \sigma+\left|P_{\lambda m} u_{1}\right|^{2}+\left\|u_{0 m}\right\|_{0}^{2}+\int_{0}^{t}\left(A^{\prime}(s) u_{\lambda m}(s), u_{\lambda m}(s)\right) d s+ \\
+ & \frac{1}{\beta} \int_{0}^{t}|f(s)|^{2} d s .
\end{aligned}
$$

From $(2.1),(2.10)$ and $(2.11)$, there exists a constant $C^{(*)}$ such that

$$
\begin{aligned}
& \left|\left(A_{2}+\lambda I\right)^{\frac{1}{2}} u_{\lambda m}^{\prime}(t)\right|^{2}+\beta \int_{0}^{t}\left|u_{\lambda m}^{\prime}(s)\right|^{2} d s+\left\|u_{\lambda m}(t)\right\|_{t}^{2} \leq C+ \\
+ & \int_{0}^{t}\left(A^{\prime}(s) u_{\lambda m}(s), u_{\lambda m}(s)\right) d s .
\end{aligned}
$$

(*) Let us denote by $C$ various constants.

It is not difficult to prove that the function $g(t)=\left\|u_{\lambda m}(t)\right\|_{t}^{2}$ is continuous. So that from Gronwall's inequality, from $V_{t} \cong V_{0}$, and from the assumption $(v)$, we conclude that:

$$
\left\|u_{\lambda m}(t)\right\|_{0} \leq C
$$

independently from $\lambda>0 m \in \mathbb{N}$ and of $t \in\left[0, t_{m}\right)$. So that, we have

$$
\left|\left(A_{2}+\lambda I\right)^{\frac{1}{2}} u_{\lambda m}^{\prime}(t)\right|^{2}+\beta \int_{0}^{t}\left|u_{\lambda m}^{\prime}(s)\right|^{2} d s+\left\|u_{\lambda m}(t)\right\|_{t}^{2} \leq C
$$

independently from $\lambda>0, m \in \mathbb{N}$ and of $t \in\left[0, t_{m}\right)$.

Therefore, from (2.12), (2.13) and by Carathéodory Theorem there exists a solution in all interval $[0, T]$.

So we obtain the following estimates:

$$
\begin{array}{cc}
\left\|u_{\lambda m}\right\|_{L^{\infty}\left(0, T ; V_{0}\right)} \leq C, \quad \forall \lambda>0, & m \in \mathbb{N} . \\
\left\|u_{\lambda m}^{\prime}\right\|_{L^{2}(0, T ; H)} \leq C, \quad \forall \lambda>0, \quad m \in \mathbb{N} .
\end{array}
$$

Where $C$ is a constant independent of $m \in \mathbb{N}$ and $\lambda>0$. From the estimate (2.14) and noting that $M$ is bounded it follows that

$$
\left\|M\left(u_{\lambda m}\right)\right\|_{L^{\infty}(0, T ; H)} \leq C, \forall \lambda>0, \quad m \in \mathbb{N} .
$$

The estimates $(2.14)-(2.16)$, imply that there exists a subsequence of $\left(u_{\lambda m}\right)$, still denoted by $\left(u_{\lambda m}\right)$, and a function $u_{\lambda}$ such that

$$
\begin{gathered}
u_{\lambda m} \rightarrow u_{\lambda} \text { weak-star in } L^{\infty}\left(0, T ; V_{0}\right) . \\
u_{\lambda m}^{\prime} \rightarrow u_{\lambda}^{\prime} \text { weak in } L^{2}(0, T ; H)
\end{gathered}
$$




$$
\begin{gathered}
\text { M.R. CLARK } \\
A^{\frac{1}{2}}(t) u_{\lambda m} \rightarrow A^{\frac{1}{2}}(t) u_{\lambda} \text { weak-star in } L^{\infty}(0, T ; H) \\
\left(A_{2}+\lambda I\right) u_{\lambda m}^{\prime} \rightarrow\left(A_{2}+\lambda I\right) u_{\lambda}^{\prime} \text { weak in } L^{2}(0, T ; H) \\
A_{1} u_{\lambda m}^{\prime} \rightarrow A_{1} u_{\lambda}^{\prime} \text { weak in } L^{2}(0, T ; H) \\
M\left(u_{\lambda m}^{\prime}\right) \rightarrow \chi \text { weak-star in } L^{\infty}(0, T ; H)
\end{gathered}
$$

The fact that $A^{\frac{1}{2}}(t) ; A_{1}$ and $A_{2}$ are weakly closed operators of $L^{2}(0, T ; H)$ was used in (2.19), (2.20) and (2.21).

\section{4 - The Nonlinear Term}

Since $H \hookrightarrow V_{0}^{\prime}$ continuously, it follows from (2.15) that:

$$
\left\|u_{\lambda m}^{\prime}\right\|_{L^{2}\left(0, T ; V_{0}^{\prime}\right)} \leq C \text {, independently of } \lambda>0 \text { and } m \in \mathbb{N} \text {. }
$$

From (2.4), (2.23) and by the compact embedding from $V_{0}$ in $H$, it follows from the Lemma of Aubin-Lions, see Lions [06], that:

$$
u_{\lambda m} \rightarrow u_{\lambda} \text { strong in } L^{2}(0, T ; H) \text {. }
$$

For $v \in L^{2}(0, T ; V)$ and $\Theta>0$ a real number, by the monotonicity of $M$ we have:

$$
\int_{0}^{T}\left(M\left(u_{\lambda}+\Theta v\right)-M\left(u_{\lambda m}\right), u_{\lambda}+\Theta v-u_{\lambda m}\right) d t \geq 0 .
$$

From this inequality, taking the limit $m \rightarrow \infty$ and using the convergences (2.22) and (2.24) we get:

$$
\int_{0}^{T}\left(M\left(u_{\lambda}+\Theta v\right)-\chi, v\right) d t \geq 0, \quad \forall v \in L^{2}(0, T ; V) .
$$

It follows, by the hemicontinuity of $M$, that,

$$
M\left(u_{\lambda}\right)=\chi
$$

By multiplying both sides of (2.9) by $\Phi \in C_{0}^{\infty}(0, T)$, integrating from $t=0$ to $t=T$, passing to the limit and using the convergences (2.19) - (2.22) we obtain,

$$
\begin{aligned}
& -\int_{0}^{T}\left(\left(A_{2}+\lambda I\right) u_{\lambda}^{\prime}, \Phi^{\prime} v\right) d t+\int_{0}^{T}\left(A_{1} u_{\lambda}^{\prime}, \Phi v\right) d t+ \\
& +\int_{0}^{T}\left(A^{\frac{1}{2}}(t) u_{\lambda}, A^{\frac{1}{2}}(t) \Phi v\right) d t+\int_{0}^{T}\left(M\left(u_{\lambda}\right), \Phi v\right) d t= \\
& =\int_{0}^{T}(f, \Phi v) d t, \quad \forall \Phi \in C_{0}^{\infty}(0, T), \forall v \in V .
\end{aligned}
$$

Since the linear combinations of $w_{1}, \ldots, w_{m}$ are dense in $D(A(0))$, it follows that the above equality, remains valid for all $v \in D(A(0))$ and for all $\Phi \in C_{0}^{\infty}(0, T)$ also. So that, $u_{\lambda}$ is a solution of the perturbed problem in the sense given in (2.6).

From this we have that

$$
\left(\left(A_{2}+\lambda I\right) u_{\lambda}^{\prime}\right)^{\prime}=-A_{1} u_{\lambda}^{\prime}-A(t) u_{\lambda}-M\left(u_{\lambda}\right)+f \in L^{2}\left(0, T ; V_{0}^{\prime}\right) .
$$

Noticing that the estimates (2.14) - (2.16) are independent of $\lambda>0$, we obtain the same convergences (2.17) - (2.22) and also the equality (2.25) replacing $u_{\lambda m}$ by $u_{\lambda}$ by and $u_{\lambda}$ by $u$.

By the above arguments, taking the limit in (2.26) we have that $u$ satisfies (2.4)-(2.6). 
From (2.6) we have,

$$
\begin{gathered}
\left(A_{2} u^{\prime}\right)^{\prime}+A_{1} u^{\prime}+A(t) u+M(u)=f \text { in } L^{2}\left(0, T ; V_{0}^{\prime}\right) . \\
\left(A_{2} u^{\prime}\right)^{\prime} \in L^{2}\left(0, T ; V_{0}^{\prime}\right) .
\end{gathered}
$$

\section{5 - The Inicial Conditions}

The proof of the initial conditions (2.7) and (2.8) are obtained by the convergences (2.17), (2.18). Let $\Phi \in C^{1}([0, T])$ with $\Phi(0)=1, \Phi(T)=0$, and $v \in V_{0}$. Then by (2.17) and using Lemma 1, with $\Phi v \in V_{0}$, we obtain

$$
\begin{aligned}
& -<\left(A_{2}+\lambda I\right) u_{\lambda}^{\prime}(0), v>-\int_{0}^{T}\left(\left(A_{2}+\lambda I\right) u_{\lambda}^{\prime}, \Phi^{\prime} v\right) d t+ \\
& +\int_{0}^{T}\left(A_{1} u_{\lambda}^{\prime}, \Phi v\right) d t+\int_{0}^{T}<A(t) u_{\lambda}, \Phi v>d t+ \\
& +\int_{0}^{T}\left(M\left(u_{\lambda}\right), \Phi v\right) d t=\int_{0}^{T}(f, \Phi v) d t .
\end{aligned}
$$

Taking the limit in the above equality, we obtain

$$
\begin{aligned}
& -<A_{2}^{\frac{1}{2}} u_{1}, v>-\int_{0}^{T}\left(A_{2} u^{\prime}, \Phi^{\prime} v\right) d t+\int_{0}^{T}\left(A_{1} u^{\prime}, \Phi v\right) d t+ \\
& +\int_{0}^{T}<A(t) u, \Phi v>d t+\int_{0}^{T}(M(u), \Phi v) d t=\int_{0}^{T}(f, \Phi v) d t .
\end{aligned}
$$

Integrating by parts $-\int_{0}^{T}\left(A_{2} u_{\lambda}^{\prime}, \Phi^{\prime} v\right) d t$, observing (2.29) and using Lemma-1, we get from (2.28) and (2.30) that:

$$
<A_{2} u^{\prime}(0), v>=<A_{2}^{\frac{1}{2}} u_{1}, v>, \forall v \in V .
$$

From this it follows the proof of Theorem 1.

Remark 1. We obtain the same Theorem 1 by considering:

$$
M: L^{2}\left(0, T ; V_{0}\right) \rightarrow L^{2}(0, T ; H)
$$

pseudo-monotone and satisfying condition (vii) (see Lions, [06]).

\section{3.- PROOF OF THEOREM 2}

If $u$ and $v$ satisfy Theorem-1, then $w=u-v$ satisfies:

$$
\begin{gathered}
\left(A_{2} w^{\prime}\right)^{\prime}+A_{1} w^{\prime}+A(t) w+M(u)-M(v)=0 \text { in } L^{2}\left(0, T ; V_{0}^{\prime}\right) \\
w(0)=0, A_{2} w^{\prime}(0)=0
\end{gathered}
$$

We'll prove that $w=0$ in $[0, T]$.

We observe that the solution $u^{\prime}(t) \in H$ and $\left(A_{2} u^{\prime}\right)^{\prime}(t) \in V^{\prime}$. Therefore it doesn't make sense the duality between these vectors. In this case, we'll use the method introduced by Visik-Ladyzenskaja [14].

For each $s$ with $0<s<T$, we'll consider the function $z(t)$ given by:

$$
z(t)= \begin{cases}-\int_{t}^{s} w(\xi) d \xi & \text { if } 0 \leq t \leq s \\ 0 & \text { if } s<t \leq T\end{cases}
$$

We have that $z(s)=0, z^{\prime}(t)=w(t)$ for $0 \leq t \leq s$ and $z(t) \in V_{0}$ for each $t \in[0, T]$. 
Defining $w_{1}(t)$ by, $w_{1}(t)=\int_{0}^{t} w(\gamma) d \gamma$, we have $z(t)=w_{1}(t)-w_{1}(s), \quad 0 \leq t \leq s$.

Taking the duality of (3.1) with (3.3) and integrating from $t=0$ to $t=T$, we obtain

$$
\begin{aligned}
& \int_{0}^{T}<\left(A_{2} w^{\prime}\right)^{\prime}, z>d t+\int_{0}^{T}\left(A_{1} w^{\prime}, z\right) d t+\int_{0}^{T}<A(t) w, z>d t+ \\
& +\int_{0}^{T}(M(u)-M(v), z) d t=0 .
\end{aligned}
$$

We have that:

$$
\begin{aligned}
& \int_{0}^{T}<\left(A_{2} w^{\prime}\right)^{\prime}, z>d t=-\frac{1}{2}\left(A_{2} w(s), w(s)\right) \\
& \int_{0}^{T}\left(A_{1} w^{\prime}, z\right) d t=-\int_{0}^{s}\left(A_{1} w, w\right) d t . \\
& \left.\int_{0}^{T} A^{\frac{1}{2}}(t) z\right) d t= \\
& =\frac{1}{2} \int_{0}^{s} \frac{d}{d t}\|z(t)\|_{t}^{2} d t-\frac{1}{2} \int_{0}^{s}\left(A^{\prime}(t) z(t), z(t)\right) d t= \\
& =-\frac{1}{2}\left|A^{\frac{1}{2}}(0) w_{1}(s)\right|^{2}-\frac{1}{2} \int_{0}^{s}\left(A^{\prime}(t) z(t), z(t)\right) d t .
\end{aligned}
$$

Substituting the above equalities in (3.4) we have:

$$
\begin{aligned}
& \frac{1}{2}\left|A_{2}^{\frac{1}{2}} w(s)\right|^{2}+\int_{0}^{s}\left(A_{1} w, w\right) d t+\frac{1}{2}\left|A^{\frac{1}{2}}(0) w_{1}(s)\right|^{2}= \\
& =\int_{0}^{s}(M(u)-M(v), z) d t-\frac{1}{2} \int_{0}^{s}\left(A^{\prime}(t) z(t), z(t)\right) d t .
\end{aligned}
$$

By using hypotheses ii), iii), v), viii) in the above equality, we obtain:

$$
\begin{aligned}
& \frac{1}{2}\left|A_{2}^{\frac{1}{2}} w(s)\right|^{2}+\beta \int_{0}^{s}|w(t)|^{2} d t+\frac{\alpha}{2}\left|w_{1}(s)\right|^{2} \leq \\
& \leq \int_{0}^{s} \mu|w(t)||z(t)| d t+\frac{\gamma}{2} \int_{0}^{s}|z(t)|^{2} d t \leq \int_{0}^{s} \mu|w(t)|\left|w_{1}(t)\right| d t \\
& +\int_{0}^{s} \mu|w(t)|\left|w_{1}(s)\right| d t+\frac{\gamma}{2} \int_{0}^{s}|z(t)|^{2} d t
\end{aligned}
$$

By applying the inequality $a b \leq \frac{\lambda a^{2}}{2}+\frac{b^{2}}{2 \lambda}, \forall \lambda>0$, in the above inequality one has:

$$
\begin{aligned}
& \left(\beta-\mu^{2} \lambda\right) \int_{0}^{s}|w(t)|^{2} d t+\left[\frac{\alpha}{2}-\left(\frac{1}{2 \lambda}+\gamma\right) s\right]\left|w_{1}(s)\right|^{2} \leq \\
& \left(\frac{1}{2 \lambda}+\gamma\right) \int_{0}^{s}\left|w_{1}(t)\right|^{2} d t, \quad \forall \lambda>0 \text { such that } \beta-\mu^{2} \lambda>0
\end{aligned}
$$

and $\frac{\alpha}{2}-\left(\frac{1}{2 \lambda+\gamma}\right) s>0$. If we choose $\lambda>0$ such that $\beta-\mu^{2} \lambda=\frac{\beta}{2}$, that is, $\lambda=\frac{\beta}{2 \mu^{2}}$ and $s_{0}$ such that $\frac{\alpha}{2}-\left(\frac{1}{2 \lambda}+\gamma\right) s_{0}=\frac{\alpha}{4}$, that is, $s_{0}=\frac{\alpha \lambda}{2(1+2 \lambda \gamma)}$, we obtain from the above equality:

$$
\frac{\beta}{2} \int_{0}^{s}|w(t)|^{2} d t+\frac{\alpha}{4}\left|w_{1}(s)\right|^{2} \leq\left(\frac{\mu^{2}}{\beta}+\gamma\right) \int_{0}^{s}\left|w_{1}(t)\right|^{2} d t
$$

$\forall s \in\left[0, s_{0}\right]$. Gronwall's inequality implies that $w_{1}(s)=0$ for all $s \in\left[0, s_{0}\right]$. Which implies $w_{1}(s)=0 \forall s \in\left[0, s_{0}\right]$, consequently $w(t)=0$ for all $t \in\left[0, s_{0}\right]$.

Using the same argument in $\left[0, s_{0}\right]$ for the Cauchy problem: 


$$
\left[\begin{array}{l}
\left(A_{2} w^{\prime}\right)^{\prime}+A_{1} w^{\prime}+A(t) w+M(u)-M(v)=0 \\
w\left(s_{0}\right)=0, A_{2} w^{\prime}\left(s_{0}\right)=0
\end{array}\right.
$$

we obtain that $w(t)=0$, for all $t \in\left[s_{0}, 2 s_{0}\right]$.

After a finite number of steps we conclude $w(t)=0$ in $[0, T]$ and the proof of the Theorem 2 is completed.

\section{EXAMPLES}

1) Let $\Omega$ be a regular bounded open subset of $\mathbb{R}^{n}$ and $H=L^{2}(\Omega), V=H_{0}^{1}(\Omega)$.

Let us define the functions $k_{1}, k_{2} \in L^{\infty}(\Omega)$ such that $k_{1}(x) \geq \beta>0$ a.e. and $k_{2}(x) \geq 0$ a.e. in $\Omega$ where $\beta$ is a constant.

We define the operators $A_{1}$ and $A_{2}$ in $L^{2}(\Omega)$ by

$$
\left(A_{1} u\right)(x)=k_{1}(x) u(x), \quad\left(A_{2} u\right)(x)=k_{2}(x) u(x)
$$

and consider

$$
A(t)=-\sum_{i, j=1}^{n} \frac{\partial}{\partial x_{j}}\left(\iota_{i},(x, t) \frac{\partial}{\partial x_{i}}\right)
$$

being the domain of $A(t)$ the space $H^{2}(\Omega) \cap H_{0}^{1}(\Omega)$ which is dense in $L^{2}(\Omega)$; where $a_{i j}=a_{\jmath i}$ and

$$
a_{i j}^{\prime}=\frac{\partial}{\partial t} a_{i j} \in L^{\infty}(\Omega \times(0, T)), \quad \forall 1 \leq i, j \leq n .
$$

Then $A(t)$ is a family of self-adjoint operators.

We also assume that:

$$
\sum_{i, j=1}^{n} a_{i j}(x, t) \xi_{i} \xi_{j} \geq \gamma\left(\left|\xi_{1}\right|^{2}+\ldots+\left|\xi_{n}\right|^{2}\right)
$$

$(x, t) \in Q, \quad 0<\gamma \in \mathbb{R}$ and $\xi=\left(\xi_{1}, \ldots, \xi_{n}\right) \in \mathbb{R}^{n} ;$ then, by Poincaré-Friedrichs inequality implies that $(A(t) u, u) \geq \alpha|u|^{2}$, for all $u \in D=D(A(t))$ and for some constant $\alpha>0$.

Noting that

$$
\left|\left(\left(A(t)-A\left(t_{0}\right)\right) u, u\right)\right| \leq \sum_{i, J=1}^{n} \int_{\Omega}\left|a_{i j}(x, t)-a_{i j}\left(x, t_{0}\right)\right| \cdot\left|\frac{\partial u}{\partial x_{i}} \cdot \frac{\partial u}{\partial x_{j}}\right| d x,
$$

being $a_{i j} \in L^{\infty}(Q)$, we have that there exists the $\lim _{t \rightarrow t_{0}}\left(t-t_{0}\right)^{-1}\left(A(t) u-A\left(t_{0}\right) u\right)$ in norm of $L^{2}(\Omega)$.

Therefore $A(t)$ is continuously strongly differentiable.

Being $A^{\prime}(t)=-\sum_{i, j=1}^{n} \frac{\partial}{\partial x_{j}}\left(a_{i j}^{\prime}(x, t) \frac{\partial}{\partial x_{i}}\right)$ with $a_{i j}^{\prime}=a_{j i}^{\prime} \in L^{\infty}(Q) \forall 1 \leq i, j \leq n$, we have $\left|\left(A^{\prime}(t) u, u\right)\right| \leq$ supess $_{Q} \mid a_{i j}^{\prime}(x, t)\|u\|_{H_{0}^{1}}^{2} ;$ where we used Cauchy-Schwarz and PoincaréFriedrichs inequalities. Then we obtain $\left(A^{\prime}(t) u, u\right) \leq \gamma\|u\|^{2}$, where $\|\cdot\|$ denote the norm in $H_{0}^{1}(\Omega) \cap H^{2}(\Omega)$.

It is well known that $H_{0}^{1}(\Omega) \cap H^{2}(\Omega) \hookrightarrow L^{2}(\Omega)$ compactly.

Let $F: \mathbb{R} \rightarrow \mathbb{R}$ be the function defined by $F(s)=s^{3}$, and $M: H_{0}^{1}(\Omega) \rightarrow L^{2}(\Omega)$ a operator defined by $(M u)(x)=F(u(x))$.

Due to the properties of $F$ it follows that $M$ is monotone, hemicontinuous bounded and

$$
\int_{0}^{t}\left(M(u(s)), u^{\prime}(s)\right) d s \geq-\sigma, \forall t \in[0, T]
$$


for all $u \in E_{c}$ where $E_{c}$ is the set $\left\{u \in L^{\infty}\left(0, T ; H_{0}^{1}(\Omega)\right), \quad u^{\prime} \in L^{2}\left(0, T ; L^{2}(\Omega)\right)\right.$ and $\|u(0)\| \leq C\}$. The constant $\sigma$ depends an $C$.

Let us prove the two last properties. Being

$$
\begin{aligned}
& |M u|^{2}=\int_{\Omega}|(M u)(x)|^{2} d x=\int_{\Omega}\left|u^{3}(x)\right|^{2} d x= \\
& \int_{\Omega}|u(x)|^{6} d x=\|u\|_{L^{6}(\Omega)}^{6},
\end{aligned}
$$

it follows from Sobolev inequalities, $H_{0}^{1}(\Omega) \hookrightarrow L^{q}(\Omega)$ with $\frac{1}{q}=\frac{1}{2}-\frac{1}{n}(n \geq 3)$. Therefore $H_{0}^{1}(\Omega) \hookrightarrow L^{6}(\Omega)(n=3)$ and, $|M u|^{2} \leq c\|u\|^{6}$. So that, $M$ is bounded.

Let $g(\tau)=\int_{0}^{\tau} F(r) d r$. Then $g(\tau) \geq 0, \forall \tau \in \mathbb{R}$, and for $u \in E_{c}$,

$$
\begin{aligned}
& \int_{0}^{t}\left(M(u(s)), u^{\prime}(s) d s=\int_{0}^{t} \int_{\Omega} u^{3}(x, s) \frac{\partial u}{\partial s}(x, s) d x d s=\right. \\
& \int_{0}^{t} \int_{\Omega} F(u(x, s)) \frac{\partial u}{\partial s}(x, s) d x d s=\int_{0}^{t} \int_{\Omega} \frac{\partial g}{\partial s}(u(x, s)) d x d s= \\
& =\int_{\Omega} g(u(x, t)) d x-\int_{\Omega} g(u(x, 0)) d x \geq-\int_{\Omega} g(u(x, 0)) d x= \\
& =-\frac{1}{4} \int_{\Omega}[u(x, 0)]^{4} d x=-\frac{1}{4} \int_{\Omega}|u(x, 0) \| u(x, 0)|^{3} d x \geq \\
& \geq-\left[\int_{\Omega}|u(x, 0)|^{2} d x\right]^{\frac{1}{2}} \cdot\left[\int_{\Omega}|u(x, 0)|^{6} d x\right]^{\frac{1}{2}} \geq-\sigma .
\end{aligned}
$$

Therefore one has studied the existence and uniqueness of solutions of the mixed problem for the equation

$$
k_{2}(x) u^{\prime \prime}+k_{1}(x) u^{\prime}+A(t) u+u^{3}=f
$$

2)In the same scheme we have analogous results for the equations

$$
k_{2}(x) u^{\prime \prime}+k_{1}(x) u^{\prime}+A(t) u+M(u)=f
$$

where $(M u)(x)=F(u(x))$ here $F(s)$ is defined by

$$
F(s)= \begin{cases}\operatorname{sign}(s) \frac{s^{2}}{1+s^{2}} & \text { if } s \neq 0 \\ 0 & \text { if } s=0\end{cases}
$$

Acknowledgments. I would like to express my thanks to Professors L. A. Medeiros and M. Milla Miranda for their encouragement and valuable suggestions.

\section{REFERENCES}

[01] Bensoussan, A.; LIONS, J .L.; PAPAniCOlaU, G., Perturbations et aumentation des conditions initials - simgular Perturbation and Boundary Layer TheorySpringer-Verlag, Lyon,(1976) pp. 10-29.

[02] CODDINGTON, E. \& LEVINSON, N., Theory of ordinary differential equations. McGraw-Hill, New York (1955) 
[03] J'ORGENS, K.,Das Anfangswertproblem im Grobem f'ur eine Klasse nichtlinearer Wellengleichungen, Math Zeitshr. 77, (1961) pp. 295-308.

[04] LARKIN, N.A., Boundary problems in the large for a class of hyperbolic equations Siberian Math. Journal, vol. 18, no 6,(1977) pp. 1003-1006.

[05] LIMA, O., Existence and Uniquiness of an Abstract Nonlinear Hyperbolic-Parabolic Equation. Applicable Analysis, vol.24, (1987) pp 101-116.

[06] LIONS, J.L., Quelques Méthodes de Resolution des Problemes aux Limites non Linéaires - Dunod, Gauthier - Villars, Paris.(1969)

[07] MEDEIROS, L.A., Non-linear Hyperbolic-Parabolic Partial Differential EquationsFunkcialaj Ekvacioj, no 23, (1978)pp. 151-158.

[08] MEDEIROS, L.A., Remarks on hyperbolic-parabolic Partial Differential Equations - 11ํㅡㄴ Seminário Brasileiro de Análise - (1978)pp. 1-12.

[09] MEDEIROS, L.A., On the initial and boundary value problem for non-linear wave equations in Hilbert space - Transl. of the Amer. Math. Soc. - Vol. 136,(1969) pp. 305-327.

[10] MELLO, E.A., Soluções Fracas de um problema Hiperbólico-Parabólico não-linear, Tese de Doutorado - IN-URFJ.(1983)

[11] MACIEL, A.B., Soluções de uma Classe de equações Hiperbólica-Parabólica com não linearidade de tipo contínuo, $23^{0}$ SBA, (1986) pp. 25-31.

[12] NEVES, B.P., Perturbações e aumento das condições iniciais de equações hiperbólica-parabólica não Linear 9ํㅡㅅㅡ, (1979) pp. 109-123.

[13] TANABE, H., Equations of evolution. Osaka University.(1977)

[14] VISIK, M.; LADYZENSKAJA, O., On boundary value problem for PDE and certain classe of operators equations. Amer. Math. Soc. Transl. Série 2, vol. 10.(1958)

[15] VAGROV, V.N., On the Cauchy for some parabólic-hyperbolic equations - Soviet. Math. Dokl, vol. 14-5, (1973) pp. 1396-1400. 


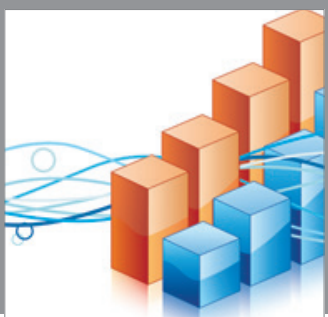

Advances in

Operations Research

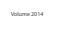

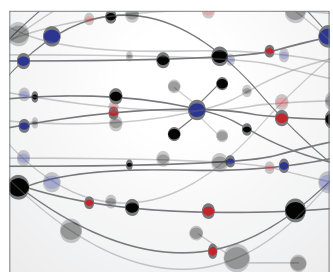

\section{The Scientific} World Journal
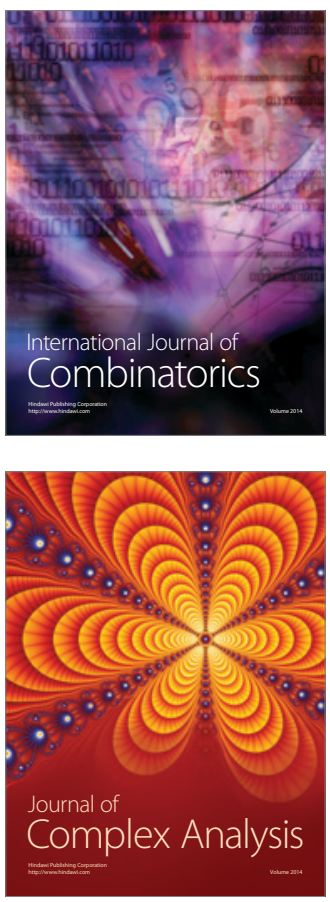

International Journal of

Mathematics and

Mathematical

Sciences
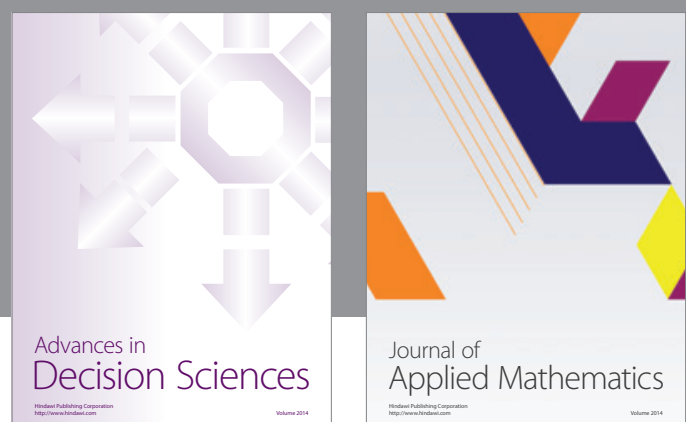

Journal of

Applied Mathematics
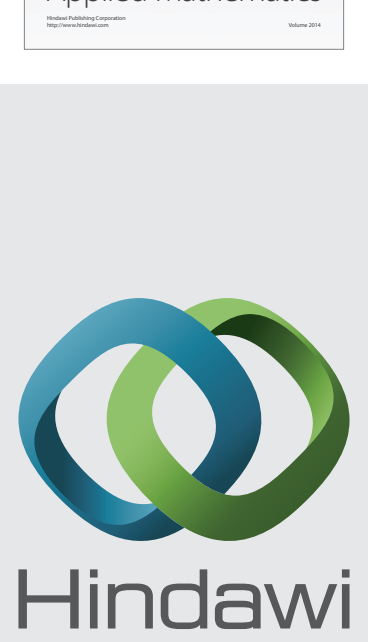

Submit your manuscripts at http://www.hindawi.com
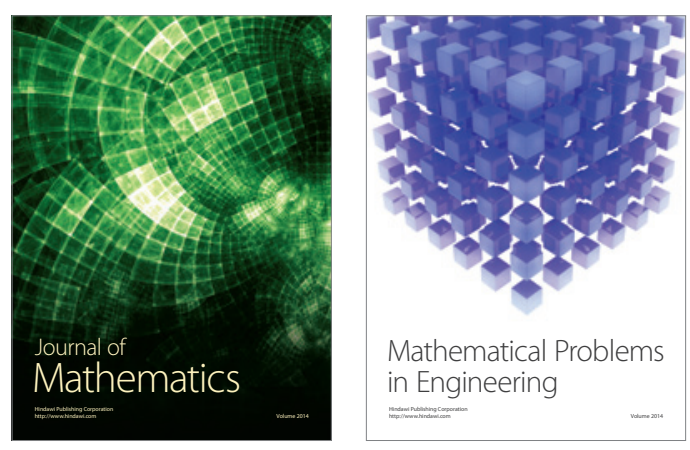

Mathematical Problems in Engineering
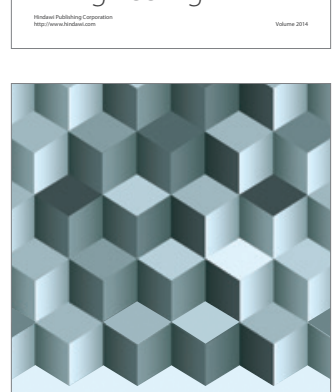

Journal of

Function Spaces
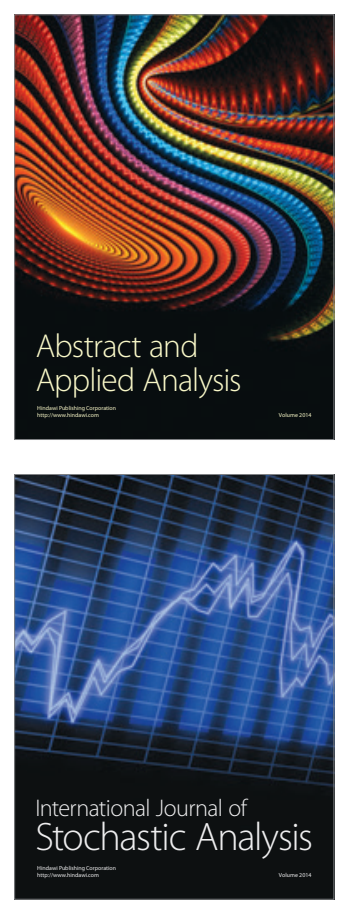

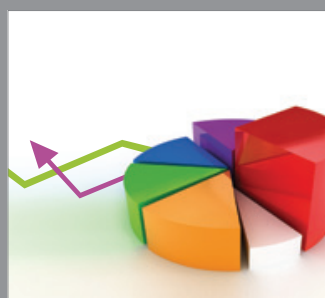

ournal of

Probability and Statistics

Promensencen
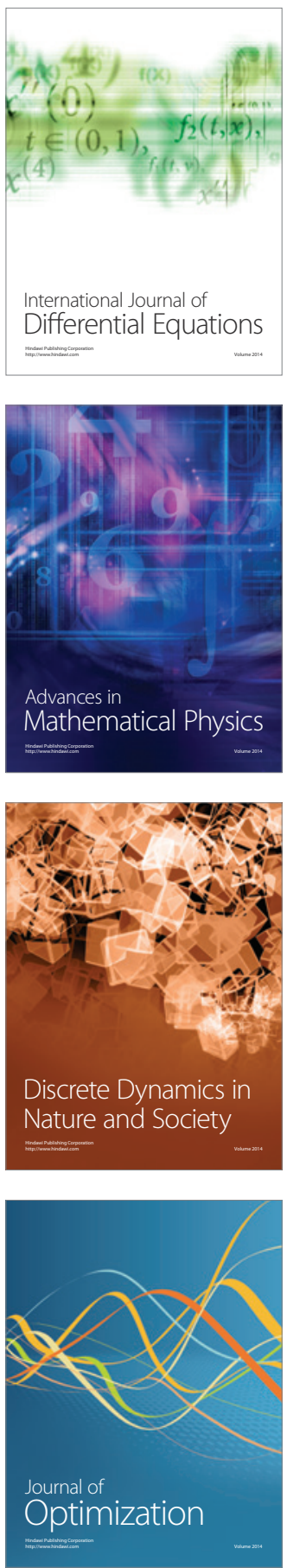